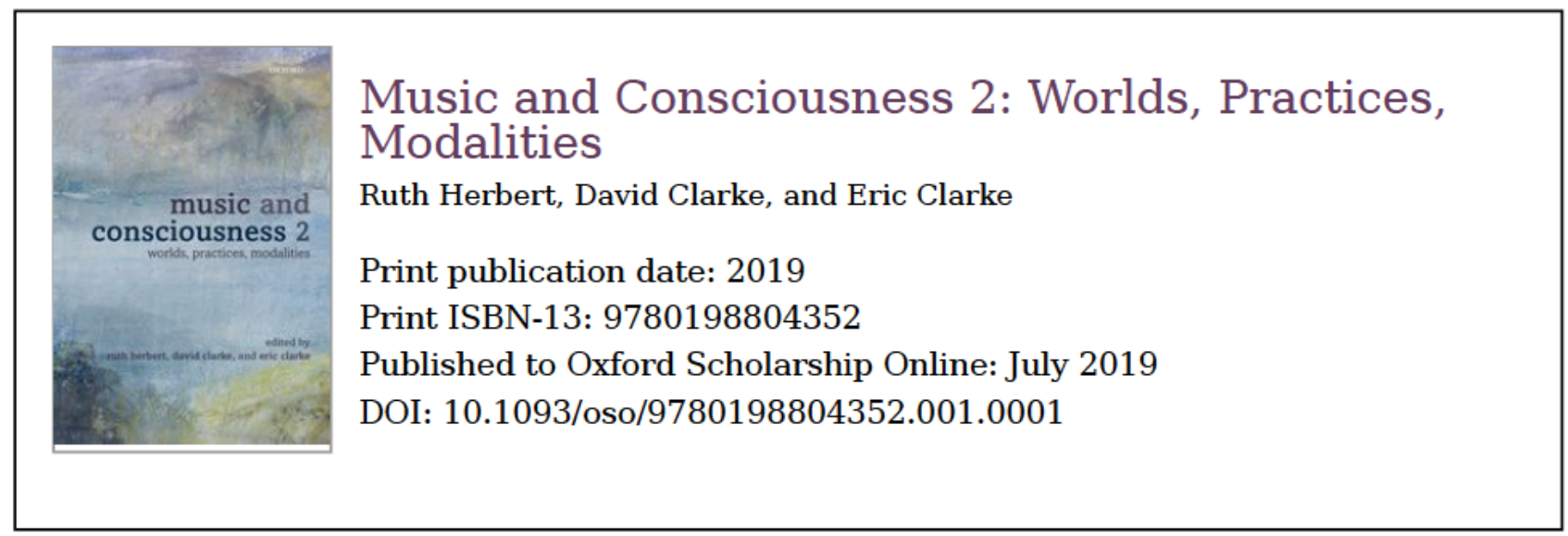

\title{
Feeling at one: Socio-affective distribution, vibe, and dance-music consciousness
}

\author{
Maria A.G. Witek
}

DOI:10.1093/oso/9780198804352.003.0006

\begin{abstract}
Keywords
In this chapter, the embodied consciousness of clubbing and raving is considered through the theory of extended mind, according to which the mind is a distributed system where brain, body, and environment play equal parts. Building on the idea of music as affective atmosphere, a case is made for considering the vibe of a dance party as cognitively, socially, and affectively distributed. The chapter suggests that participating in the vibe affords primary musical consciousness - a kind of pre-reflexive state characterized by affective and bodily knowledge-and speculates about the neural correlates of clubbing and raving by means of an analogy with brain research on psychedelic states.
\end{abstract}

Keywords: dance music, clubbing, raving, vibe, extended mind, psychedelic

\section{Introduction}

I was at 'Unity II' rave this past weekend, and I had the most wonderful experience. As the dj played his trance set I could feel the energy come out of the speakers, diffuse into the souls and minds of all the dancers and then reflect back out into the room. It was the most incredible, almost overwhelming, feeling of love and tranquility I have ever experienced. I looked at the others around me and I could see in their eyes the very peace and warmth that I was feeling. Their gestures and the way they danced only amplified this 'vibe' and continued it through the night, and I wondered if we were all living in a dream ...

\section{PLUR}


keep the vibe alive!!!

P.M. West (1996) on hyperreal.org

The opening description was posted on hyperreal.org, a website that among other rave-related material (such as photo and flyer galleries, and information on music, DJs, labels, and drugs) features a series of 'rave testimonials' from the 1990s. This decade saw the rise of a youth subculture whose members spent their weekends in abandoned warehouses or fields where sometimes thousands of people would dance until the early morning to electronic dance music such as house, techno, trance, and drum-and-bass. After a legal crackdown in the 1990s, rave developed into a club and festival scene, which remains a huge musical subculture and night-time economy. Some nightclubs, such as the famous Berghain in Berlin, have acquired an international cult reputation and have been given a tax status as a 'cultural venue' on a par with classical concert halls (Oltermann 2016). Other clubs have faced revoked licences and permanent closure due to zoning, gentrification, and concerns about drugs (Garcia 2016). In the UK, there has been a rave renaissance (Gilmour 2016), and regardless of official cultural or moral stances, dance music culture has become a global musical phenomenon, with world-famous DJs playing every weekend in virtually every big city in the world.

(p.94) P.M. West's post on the hyperreal.org website is a typical example of how ravers describe raving and clubbing. The recognition of one's own emotions in the eyes of the surrounding dancers vividly conveys the sense that the affective experience of the rave is shared among the ravers. West describes the energy travelling between the speakers, the dancers' souls and minds, and the space that surrounds them, connecting their bodies, the music, and the environment. The rave mantra 'PLUR' (standing for Peace, Love, Unity, and Respect) encapsulates the emotions they share, echoed in the name of the rave, 'Unity II'. West attributes the diffused energy to the 'vibe' of the rave, a force that needs to be 'kept alive', and which the dancers feed with their movements, amplifying its intensity and duration, leading West to wonder if they have entered a different kind of consciousness-more like a dream than waking reality. 
This raver's reflection resonates well with ethnographers' accounts of dance music cultures, and many of the emerging themes in these accounts-collective emotions, unity, distributed energy, vibe, and altered states of consciousnessappear frequently in cultural and sociological studies. Such studies focus largely on how these experiences relate to identity politics (Fikentscher 2000; Lawrence 2011; Rief 2011), youth culture (Thornton 1995), and political resistance-or the lack of it (Gilbert and Pearson 1999; Jordan 1995). To complement these studies, this chapter takes a phenomenological approach to dancing, clubbing, and raving as a form of embodied consciousness, and considers how the neurofunctional architecture of the brain might contribute to such modes of consciousness. It looks at how this musical activity is cognitively, socially, corporeally, and emotionally distributed in the affective atmosphere of the vibea force field that is co-determined by the beats and sounds of the music, the dancers' gestures and emotional expressions, the DJ's dynamic performance, the visual effects and temperature of the environment, and often-but not alwaysdrugs, such as MDMA, alcohol, and psychedelics. The vibe is considered as a non-anthropocentric force where subjectivity is not just shared between people but is diffused across a greater, and partially non-human whole. Participation in this vibe affords a kind of pre-reflexive consciousness in which affective and bodily knowledge trumps analytical and reflexive knowledge. Finally, the chapter speculates about the neural make-up of a dance-induced ecstatic brain by analogy with neuroimaging research on the effect of psychedelic drugs.

Distributed mind: Cognitive, social, and affective extensions 
The blurring of subject-object distinctions in cognition is a central argument in Extended Mind Theory (EMT), first proposed by Andy Clark and David Chalmers (1998; see also Clark 2008). Often viewed as the most extreme of the 4Es (situated views of the mind referred to as Embodied, Embedded, Enacted, and Extended; see Rowlands 2010), EMT rejects the view that mental processes happen exclusively within the confines of the skull, asserting instead that these processes extend across the brain, the body, and the environment. Cognition is not just connected to the outside world through active engagement and (p.95) bodily sensation: there are no fixed boundaries at all between the brain, the body, and the environment. The relationship between the mind and the environment is malleable and dynamic, and cognition is thus fundamentally redefined as a system in which brain, body, and world play equal parts. The external objects that we use in cognitive processing are not simply tools but are fully part of the cognitive process itself. According to the widely cited 'parity principle' (Clark and Chalmers 1998), external objects are to be understood as components of cognitive systems when they function indistinguishably from processes taking place in the brain. ${ }^{1}$ A smartphone, for example, with inbuilt functions such as diary, contact book, internet browser, and calculator, performs some of the mental work that is needed to remember an appointment, a phone number, or a historical fact, or to perform a mathematical calculation. The phone is functionally equivalent to a part of our brain when we use it, and is therefore properly understood as a component of a distributed mental system.

While EMT was originally a cognitive theory (Clark 2009), some have since then taken a more liberal view and have argued that the mind can be extended in other, more subjective ways. Shaun Gallagher's understanding of EMT (2011, 2013) goes beyond objects to include other subjects and groups of subjects, with socially distributed minds being exemplified in the legal system, scientific research, and the family. Gallagher asks us to consider a court case as an example in which judgements are not confined to the brains of individuals, but are distributed across judges, jury members, lawyers, experts, legal texts, cultural practices, and societal norms. Importantly, such judgements could in fact not be made solely inside the heads of individuals, and so the cognitive work is by definition extended and not just embedded: 'the particular cognitive process of forming a legal judgment, is what it is only in that [legal] system' (Gallagher 2013: 7). Gallagher specifies a more enactive than functionalist approach to cognitive distribution, and emphasizes that what is extended is not the actual materials of the brain, but rather its cognitive and social processes. 
EMT was also initially claimed to exclude extensions of conscious experience (Clark 2009), but more recently some have argued that emotions can also be distributed (Stephan et al. 2013). On the premise that cognition and emotion cannot be separated, Jan Slaby (2014) argues that our embodied engagements with the world are inextricable from our emotional experience of it, and he thus situates emotions within the EMT framework. By definition, emotions are relational-they are towards something: a landscape, a lover, our self-image, or a piece of music-with the consequence that these scenes, things, events, or people to which the emotions relate must be seen as part of a distributed affective system. Slaby (2014), like Gallagher (2013), argues that this relational rather than locational definition of embodiment brings EMT closer to enactivist theories: enactivists (p.96) argue that perception and cognition are processual because they are forms of active engagement with the environment (Thompson 2010). Thus, seeing emotions as a reciprocal active process between organism and world renders the question of 'where' emotions are located-whether inside or outside the brain-irrelevant. Slaby introduces the notion of 'phenomenal coupling' to 'define the direct, online engagements of an agent's affectivity with an environmental structure or process that itself manifests affect-like, expressive qualities' (2014: 41), and he lists music as one such example.

Joel Krueger (2013) (see also this volume, Chapter 3) argues that music offers particularly powerful ways of extending our emotions. Many people use music to regulate their emotions and identity, as captured in Tia De Nora's characterization of music as 'a technology of the self' (DeNora 2000). Krueger proposes that music acts as 'affective scaffolding', allowing us to 'offload' some of the regulatory processes involved in our emotions onto the dynamics, rhythmic structures, harmonic progressions, and lyrics of music. One of the core principles that enables this kind of musical extension of emotions is entrainment -the process by which two independent rhythmic processes are coupled to achieve a degree of synchrony (Jones 2009; Phillips-Silver et al. 2010; Trost et al. 2017). As Krueger puts it, we engage with music by 'synchronously organizing our reactive behavior and felt responses' (2013: 4), giving rise to musically extended emotions that are more complex and powerful than those confined to the brain and body. Like music, other people can act as scaffolding for our emotions (Krueger 2015). When two or more individuals relate to each other through coordinated actions, intentions, and beliefs, emotional experiences and their regulatory processes can be interpersonally distributed. Here again it is the entrainment between two processes-in this case between two people-that provides the basis for interpersonally converging emotions. As in his account of music, Krueger sees shared emotions as special because their joint construction gives access to phenomenological experiences different from those that we experience on our own. 
But can emotions be shared beyond dyads, and what are the conditions for an emotion to be shared? Von Scheve and Ismer (2013) propose a bottom-up theory of how collective emotions come about and how they are shaped and embedded in culture. Collective emotions are defined as 'synchronous convergence in affective responding across individuals towards a specific event or object' (Von Scheve and Ismer 2013: 406). Here and elsewhere (Von Scheve and Salmela 2014), the sociologist Emile Durkheim is cited as providing the first focused analysis of what he called 'collective effervescence' (Durkheim 1912/1995). He observed that when people congregate in rituals, 'a sort of electricity is generated from their closeness [which] quickly launches them to an extraordinary height of exaltation. Every emotion resonates without interference in consciousnesses that are wide open to external impressions; each echoing the others' (Durkheim 1912/1995: 217, cited in Von Scheve and Salmela 2013: xiv).

Von Scheve and Ismer (2013) propose that the face-to-face interaction of people in such gatherings affords shared emotions via motor mimicry and emotional contagion. Through synchronized gestures and joint attention, the emotional expressions of each agent are infectious and become mirrored both in the other agents' bodily expressions (p.97) and in their subjective feelings. But it is not just the proximity of people that affords shared emotions. Through shared exposure to cultural knowledge in norms, beliefs, and values, people have a tendency to respond to a given event in emotionally similar ways. Furthermore, people can experience emotions purely on the basis of the specific social groups to which they see themselves as belonging. No face-to-face interaction need take place in order to share an emotional disposition, such as the love of heavy-metal music or an aversion to bubblegum pop music; but when people share an actual physical space, such shared emotions can be amplified. 
If the mind can be extended cognitively, socially, and affectively, then we might ask whether the EMT hypothesis has any practical limits. Are there any situations or aspects of the mind that are entirely confined to the brain? Some might argue that dreams are inside the head, because an individual is not actively engaging with their environment during sleep. But the fact that external surroundings can sometimes be represented in dreams-as when the sound of an alarm clock appears in the dream as someone's incoherent shoutingchallenges the idea that dreams are entirely intracranial (see also Windt 2017). There is more disagreement, however, about the extendedness of consciousness itself. In an ongoing discussion, cognitive philosophers are developing new answers to the question of whether the 'vehicles' or 'circuitry' of consciousness are distributed into the environment or confined to the brain. Andy Clark is a proponent of the latter argument $(2009,2012)$, insisting that while the contents of conscious experience can be extraneural, its machinery is entirely explained by computational (specifically predictive) processes in the brain. Dave Ward (2012) disagrees, on the basis of the fundamentally enactivist assertion that experience should always be understood as an interaction between individuals and their environments. For Ward, accepting the extraneural distribution of consciousness is simply the logical result of an enactive understanding. If experience is fundamentally processual and interactive, then the machinery of the different contributors to the interaction-both in the individual and in the environment-must play fundamental roles in what defines the experience. However, whether we are aware of the extensions of our experiences or not is a separate question. As is argued in this chapter, dance-music vibes are neither more nor less extended than other experiences: they are simply experiences in which the distribution of cognitive and affective processes across mind, body, and environment becomes both mentally and physically noticeable.

Affective atmosphere and smooth space 
The collective effervescence that Durkheim described suggests that shared emotions can have a dynamic physicality. When people gather together and engage in a shared activity-be it sitting in a courtroom quietly observing a gruelling court case, or jumping and shouting vigorously in a sports stadiumthey can feel a certain affective atmosphere. To Slaby (2014), affective atmospheres exemplify the phenomenal coupling that occurs between an affective agent and an environment expressing affect-like qualities. The phenomenologist Hermann Schmitz states that 'emotions are atmospheres poured (p.98) out spatially' (Schmitz et al. 2011: 255). In his radically antidualist approach, the sensing and emoting body is posited as a felt surfaceless space, one that is not measurable or locational, but that exists in embodied experience. As surfaceless atmospheres, emotions exist in public space and are confined to neither body nor mind. Schmitz describes them as 'half-entities' in which cause and effect are one and the same, and duration can be interrupted; they are like 'the wind, voices, the sense of overpowering gravity, electric shocks, pain, melodies and many sounds such as a shrill whistle or an earpiercing noise, night, time, especially if it seems to become unbearably long in boredom or tense anticipation' (Schmitz et al. 2011: 256).

Schmitz's notion of emotions as atmospheres assumes comparable importance in Friedlind Riedel's study of the Sunday worship services of the Closed Brethren Assemblies in Germany (2015). Riedel argues that the apparent ineffability of music-in worship services but also in social music events more broadly-comes from its multisensory rather than purely auditory attributes. In such situations, music is to be understood not simply as performance but as atmosphere (Riedel 2015: 95). Being a musical atmosphere, the affectivity of worship is diffused across the minds and bodies of the worshippers, the vocal sounds of the hymns, the reverberations, lights, and scents of the church, and the Holy Spirit that is believed to be present. For the participants, the liminality of the atmosphere affords a 'new' experience in the form of spiritual renewal and proximity to God. 
Riedel relates the idea of affective atmosphere as surfaceless space to Deleuze and Guattari's (1987) notion of 'smooth space'. In contrast to 'striated space', which is a 'metered space initiated by stratification, hierarchisation and centralisation, productive of dichotomies and static identities' (Riedel 2015: 103), ${ }^{2}$ smooth space is a space of intensities that do not correspond to delineated objects and subjects, and where feelings and events exist as constellations of things, movements, and processual 'becomings' (2015: 103). Riedel argues that smooth space can transform into striated space through repetition, inscribed in the musical repertoire and engendered in the power relations of the Church. When certain hymns are performed on a weekly basis, their atmospheres become idealized and canonized, contributing to the establishment of doctrine. Nonetheless, in the process of becoming within the smooth space, subjectivity and affectivity remain indeterminate. And because atmosphere is a 'half-thing', one cannot separate cause from effect or agent from action, echoing enactivist theories of cognition and perception (Thompson 2010). In the worship of the Closed Brethren, 'the attendees perceptually become "Children of God" and "brothers" of their "Lord Jesus Christ" ' (Riedel 2015: 100); but because there is no achievable end-goal in the musical and affective atmosphere, becoming is endless and the worshippers know that they will never actually reach sainthood.

(p.99) The feeling of dancing in a nightclub or at an outdoor rave is clearly very different from that of quiet religious worship. ${ }^{3}$ Yet the idea of smooth space has also provided dance-music researchers with a way of understanding the liminality of raving and clubbing. In Tim Jordan's analysis (1995), raves are described as 'vast celebrations [where] participants gradually lose subjective belief in their self and merge into a collective body' (1995: 125). The smooth space of raves is where the 'desiring-production' of the rave is transformed into a 'desiring-machine' (Deleuze and Guattari 1987), an assemblage of music, dance, drugs, and lights where ravers strive for a desubjectified state of rapture and euphoria. Making reference to another of Deleuze and Guattari's conceptsthe 'body without organs' (BwO), in which organ functions are not fixed (Deleuze and Guattari 1987)-Jordan writes:

The $\mathrm{BwO}$ of raving is the undifferentiated state that supports the connections that the rave-machine makes between its different elements. This undifferentiated state is a collective delirium produced by thousands of people jointly making the connections of drugs to dance, music to dance, dance to drugs, drugs to time, time to music and so on, and thereby gradually constructing the state of raving and so the $\mathrm{BwO}$ of raving. The delirium is non-subjective and smooth, as all the connections and functions of the machine give way to simple intensities of feeling. (Jordan 1995: 130) 
Just as the surfacelessness and ineffability of congregational music afford the affective atmosphere of religious worship (Riedel 2015), so does the smoothness and lack of differentiation of dance music offer the collective delirium of raving (Jordan 1995). The smoothness of these experiences is largely due to the indeterminacy of their various components, a point to which I return.

Vibe and embodied distribution in clubbing and raving At raves and in clubs, the affective atmosphere is referred to as the 'vibe' (Fikentsher 2000) - a term that ravers and clubbers use frequently and which appears in several of the rave testimonials on hyperreal.org. One raver writes on the website: 'When you open your heart, and trust the whole group you dance with; when you feel love with everyone, and they return it, a higher vibration can be reached. This happens when a crowd is deep into the vibe of House.' The vibe is collectively shaped by a range of elements, such as the DJ and the music that they are playing, the dancers and their style of movement, the particular space of the club or the landscape (in the case of outdoor raves or festivals), the sound level, the tactile vibrations of the bass, the tempo, the lighting, the temperature, the smell, and the fashion. While some of these elements remain more or less stable throughout the night (the space and the sound level), many of them fluctuate and are dynamically transformed on a minute-by-minute or hour-by-hour basis (the changing of DJs, arrival of new clubbers, rising heat). 
(p.100) As the previous rave testimonial makes explicit, vibe is short for vibration-which points to an important aspect of the affective atmosphere of clubbing and raving that sets it apart from many other kinds of affective atmospheres, such as that of the Closed Brethren described by Riedel (2015). The vibe has a distinctive rhythmic quality, as acknowledged by Sally Sommer, who defines vibe as 'an active communal force, a feeling, a rhythm that is created by the mix of dancers, the balance of loud music, the effects of darkness and light, the energy. Everything interlocks to produce a powerful sense of liberation. The vibe [is] an active, exhilarating feeling of "now-ness" that everything is coming together-that a good party is in the making' (Sommer 2001: 73). Rhythm is also central in Kai Fikentscher's account of 1990's dancemusic culture in New York, where it is conceived as a 'synchronising force, facilitating the process in which a vibe is created, shaped, and maintained' (Fikentscher 2000: 80). First and foremost, it is the rhythmically regular beat that binds the DJ, the music, and the dancers together. Keeping the beat going, through careful choices of records and beat-matching, is a defining goal for the DJ-just as it is also the task of the dancers to keep the beat, irrespective of stylistic differences in the ways in which clubbers dance to different kinds of music. The light shows at dance clubs are also often rhythmical, further contributing to the rhythmicity of the vibe. But it is not just simple synchronization between these different modalities of rhythm that makes the vibe: it is the particular ways in which the rhythms in dancers' movements, the music, and the lights interlock that give the vibe its affective character.

I have argued elsewhere (Witek 2017) that there is a special rhythmic quality in electronic dance music, such as house and techno, that particularly facilitates this interlocking of music and dancers. In these musics, and in groove-based music more generally, the rhythms are frequently syncopated, often to the extent that they form polyrhythms. These syncopations generate gaps in the rhythmic surface, and the desire to move that we feel with syncopated groove-based music is our body's response to the invitation to fill these gaps, by synchronizing and moving to the beat. In this way, dancers actively participate in the musical structure and literally enact the beat of the music-a powerful example of the musically extended mind and body. In becoming part of the music by filling in the gaps when we dance, we dissolve the boundaries between our minds, our bodies, and the music. Even when we listen without moving our bodies, we fill in the gaps virtually, with our embodied attention. 
But it is not just the temporal structure of the beat that facilitates embodied and social distribution. Luis-Manuel Garcia (2015) explains how the texture and granularity typical of electronic dance-music production gives the music tactile and haptic qualities that afford a kind of sensory-affective bridging between touch, sonic experience, and connectedness on the dance floor. The direct impact of the beat, caused by high-amplitude bass frequencies and sharp attack envelopes, can be physically felt in the body with most dance club sound systems. Furthermore, many of the sounds used in house and techno have a distinctly bodily or 'fleshy' quality, often as a result of the direct sampling of body sounds (the most common of which is hand claps). Finally, the granularity of much electronic dance-music sound production facilitates experiences that index the sense of touch: by (p.101) manipulating their microtemporal structure, for example by smoothing and sustaining their decay, sounds can be heard as having a sonic grain that is cross-modally associated with a particular feel to the touch. This multisensory experience of tactility in electronic dance music adds to the distributed sense of sociability and blurring of boundaries between dancers/listeners and music because, as Garcia points out, 'touch is an inherently interactive mode of perception' (2015: 71).

Elsewhere, Garcia makes explicit both the metaphorical (as atmosphere or affect) and literal meaning (as physical force) of vibe and vibration in electronic dance music (Garcia, in press). Based on interviews with dancers and performers in the Berlin dance-music scene, he lays out an 'ontology of vibration and resonance' in electronic dance music that links affect to collective experience. The sociability and affectivity of the vibe is mediated by synchronously vibrating, resonating with, or attuning to the music-especially at the physically felt bass frequencies: 'vibrational affect in the form of sound waves fill the surrounding space and reverberate back to the sound-source, affecting the self but also folding the external world into relations of absorption, reflection, and complex interference' (Garcia, in press). 
There are plenty of other phenomenological descriptions in ethnographic and cultural studies of clubbing and raving that are consistent with the idea of affectively and socially extended minds. An explicit parallel is found in Silvia Rief's work on dance-club identity politics, which presents clubbing as a 'prosthetic culture'-a term that 'refers to a new technique of self by means of experimentation, through which aspects previously deemed immutable, fixed and automatic are brought into the realm of volition, conscious calculation and intentional modification ... [P]rosthetic culture is ... about self-extension' (2011: 97). Similarly, Ben Malbon (2002: 108) describes 'the sensation of oneness and the liminality of self/wider group that can be experienced within apparently diverse dancing crowds', and reflects upon the shared experience with his fellow clubbers of 'want[ing] the music to take us over; to become us in some way' (Malbon 2002: xii). He quotes one of his informants as saying that in the dance club, 'you feel that you're the conductor-you ARE the music' (Malbon 2002: 91). Malbon describes experiences of 'being somehow taken outside of or beyond oneself-especially while dancing', invoking Freud's description of 'oceanic' experiences: 'a feeling of an indissoluble bond, of being one with the external world as a whole' (Freud 1961: 65, cited in Malbon 2002: 107). Hillegonda Rietveld observes that at raves, 'egos melt in the sweltering frenzied heat of the mass of sweating bodies' (Rietveld 1993: 63), to which Jordan adds that 'ravers may no longer notice the lights, the other ravers or the music as separate elements but feel them as one intense event' (Jordan 1995: 130). From these and similar statements, it is apparent that the blurring that is experienced at raves and in clubs is not just between dancers' individual subjectivities, but also between their subjectivities and the sounds of the music as well as other multisensory stimulations such as temperature, visual effects, and drugs. 
As Krueger $(2013,2015)$ has argued, it is likely that entrainment provides an important cognitive and sensorimotor mechanism in enabling affective and social extension. Even when the rhythms of electronic dance music are syncopated, listeners/dancers (p.102) are expected to perceive the beat and successfully synchronize to it through the coupling of their attentional processes to the implied metrical framework. However, in raving and clubbing, the role of entrainment is not only to facilitate the offloading of dancers' emotions onto the music. Rather, ravers and clubbers become part of a greater distributed affective system, a 'half-thing' like the affective atmospheres described by Schmitz, in which cause and effect are blurred (Schmitz et al. 2011). The vibe is not just the extension of an emotion from subjects to the music to which they are listening or dancing-although such limited subject-to-music extensions are certainly possible. The vibe is distributed across a much greater variety of external objects and other subjects-indeed, it does not originate in an individual dancer in the first place. The vibe is a collective energy that can quickly be transformed as a result of both subtle and dramatic changes in one or more of the contributing elements, such as when the DJ changes to a more ambient record, or when a group of clubbers enters the dance floor looking for trouble.

Social media reports of a particularly legendary night at one of the world's most famous clubs illustrate this kind of change. Berghain is a Berlin nightclub in a converted power plant, which first opened as a gay club (Garcia 2011). Despite subsequent changes in its audience due to its national and international reputation, it is still associated with liberated sexuality. Parties at Berghain will often last from Friday until Monday, adding to the timelessness of the vibe. There are two main dance floors-one a cavernous open concrete room, the other, the Panorama bar, a more intimate space; and there are many smaller spaces, passageways, and a chill-out area. All of the spaces are either pitch dark or dimly lit (although during the daytime, daylight will inevitably filter through the building's large windows, some of them stained glass), and the dance floors are lit with moving, often rhythmical and colourful light. On this particular night, there was a thunderstorm, and lightning struck the building, with interesting consequences for the vibe. Dustin Zahn, one of the DJs who played that night, describes the events: 
The club was packed during the hottest day of the summer but around 22:45, a storm came through and lightning struck Berghain ... The emergency power kicked in, which turned on every light in the house and killed the air conditioning. For 5 hours, the lights remained on and the place kept getting hotter with people ramming the dance floor going crazy to [DJ] Pär Grindvik's now legendary set. 10 minutes before I took over, they fixed the light situation and the crowd went crazy. With the lights off, a new vibe took over and $3 / 4$ of the dance floor was topless. There was no separation of class, sex or race: just skin, dancing and great, positive vibes. During all my sets, I've never seen anything like this from the Berghain DJ booth before. (Quoted in Karwowski 2016)

The atmospheric lighting of Berghain is normally an important constituent of the vibe, which the harsh emergency lighting would be expected to attenuate rather than amplify, though apparently not in this case. Rather, the emergency lights added to it, almost as if the storm-the electricity of the lightning-had energized the vibe into an even more intense level of collective effervescence, to use Durkheim's term (1912/1995). When the emergency lighting was finally replaced by the usual club lights, the vibe was further amplified, reaching the deindividuated state that the DJ describes. As this singular event demonstrates, there is no fixed recipe for the vibe. It can never be fully controlled by any one (p.103) person, event, or circumstance, but is a collective energy, in which specific elements may influence it in particular ways (for example the lighting), but which may be displaced-unpredictably and temporarily in this case-by totally unanticipated developments (the lightning).

The primary consciousness of clubbing and raving 
Even if the origin of the vibe is non-anthropocentric, the vibe is of course subjectively experienced by everyone present in the club-though not necessarily all the time or in the same way. And this subjective experience is afforded by the social, affective, and cognitive distribution of the vibe. Eric Clarke (2011) suggests that when we listen to and engage with music, we can experience a kind of musical consciousness that is set apart from our normal waking consciousness. He draws on the distinction between primary and higherorder consciousness made by Antonio Damasio (1999) where primary consciousness is 'roughly equivalent to what is in an organism's current awareness, or the contents of its perceptual present' (Clarke 2011: 194). ${ }^{4}$ Primary consciousness depends upon the organism's sensorimotor engagement with the world, and is largely non-verbal and behavioural-a kind of bodily and proprioceptive knowing. Higher-order consciousness, in contrast, is largely verbal and 'brings with it the capacity to be aware of, and reflect on, a past and a future-most specifically the narrative of events that constitutes a person's autobiographical self' (Clarke 2011: 195). In response to Edelman's (1989) claim that higher-order consciousness cannot be suppressed once acquired, Clarke suggests that intense musical experiences provide a way for the subject to temporarily bypass or 'silence' higher-order consciousness and recapture such primarily corporeal and affective states. He cites dancing to music as one such intensely physical and affective musical experience that may give access to primary consciousness. Clubbers and ravers can clearly reach the kinds of highintensity experiences that bypass reflexive and discursive awareness, commonly reporting states of 'ecstasy', 'timelessness', and 'eternal present' (Malbon 2002; Rief 2011).

However, as Malbon emphasizes, clubbers and ravers are not in a trance. ${ }^{5} \mathrm{He}$ insists that the 'exstasis' that is experienced never completely eliminates the agency of dancers, and that in 'working together' on the dance floor they gain 'a greater control and power through the loss of control' (Malbon 2002: 114). In other words, the cognitive, affective, and social distribution of the vibe can also produce intensely personal experiences:

Some control of the body is ceded to the music and the crowd, yet through this semi- or unconscious ceding a heightened sense of control over the body can be established. The apotheosis of this (p.104) phenomenon is that clubbers can lose a sense of themselves as entities separate from the crowd-they feel lost in the crowd-yet they can use this sense of merging and harmony with the crowd as a context for interpretation and contemplation of self. (Malbon 2002: 114) 
Thus, the primary musical consciousness of clubbing and raving provides the building ground for the various performances of identity and sexuality with which this subculture is associated (Fikentscher 2000; Gilbert and Pearson 1999; Rief 2011; Thornton 1995). When the vibe provides the right conditions, dancers can be both 'lost and found' in music (Clarke 2014).

As Damasio and Edelman recognize, primary consciousness remains present in the background during normal waking consciousness; higher-order consciousness does not eliminate other more embodied and immediate forms of knowing and engaging with the environment. But when we consciously and deliberately reflect on (or speak of) our own experiences, this primary mode of consciousness may recede from our awareness. And while intensely listening to, dancing to, and otherwise engaging with music may allow people to experience a state of primary consciousness in which the mind and body are cognitively, socially, and affectively distributed, such extended notions of the self are not excluded from situations in which higher-order consciousness dominates our awareness. Extended mind theory does not only apply to out-of-the-ordinary experiences like clubbing and raving: it is a theory of how the mind works all the time. The mind is always extended when we listen to music, but when our musical experiences are intensely engaging, such as when clubbing and raving, that extension can be more palpably felt. Primary consciousness is the level at which we directly experience our 'selves' as wrapped up in the world, rather than in the more mediated, reflective, and self-conscious condition of higherorder consciousness. Clubbing, raving, and other 'oceanic' experiences are special because they put us more in touch with this condition and help us realize that what we understand as 'self' is always already partly extended.

Neural correlates of primary consciousness 
What, then, is happening to individual dancers when they experience themselves as extensions of the vibe? Are out-of-the-ordinary 'oceanic' experiences characterized by out-of-the-ordinary neurophysiological functioning in the organism? If the smooth space of an affective atmosphere like the vibe encompasses the mind as much as the body and the environment, then what is happening in the brain when the primary level of consciousness comes to the fore? While it is currently next to impossible to observe what happens in the brain when we experience the affective atmosphere of dancing ${ }^{6}$-not only because of the restrictions on movement inside most brain scanners but also due to the difficulty in reconstructing something as complex and indeterminate as vibe in a laboratory setting - there are other 'oceanic' experiences that lend themselves more easily to (p.105) neuroscientific investigation, and that can offer instructive parallels. One such 'oceanic' experience which has recently come under increasing scrutiny is the psychedelic state. In what follows, the extent to which the affective experiences of raving and clubbing might be mirrored by the affective experiences induced by psychedelic substances will be considered, followed by a review of some functional neuroimaging research using psychedelic drugs to study primary consciousness. This neuroimaging research suggests that the spatiotemporal complexity-specifically the criticality, metastability, or entropy-of neural network dynamics may be significant in explaining primary consciousness and extended mind.

Psychedelics are a collection of psychoactive drugs whose active ingredients occur naturally in certain plants or mushrooms (e.g. psilocybin mushrooms) or artificially in some designer drugs (e.g. LSD). ${ }^{7}$ Their effects include perceptual changes (visual pseudo-hallucinations, illusions, and synaesthesia), cognitive changes (increased sense of meaning), affective changes (feelings of transcendence, grief, joy, and anxiety), and changes in subjectivity (feelings of ego-dissolution) (Griffiths et al. 2006). The most obvious association between psychedelics and raving and clubbing is that many clubbers and ravers use psychedelic drugs to enhance their experiences (Palamar et al. 2017). It has been suggested that the ecstatic experiences associated with clubbing and raving are often short-lived and fleeting unless drugs are involved (Malbon 2002). Although psychedelics are used, the more common drug in rave and clubbing culture is Ecstasy, whose main active ingredient is MDMA (Palamar et al. 2017). This drug is reported to prolong and intensify the 'oceanic' experiences that dancing to house and techno music affords (Malbon 2002, 109). MDMA elicits intense experiences of euphoria, emotional warmth, openness, and empathy towards others (Bedi et al. 2009; Dumont and Verkes 2006). It is often considered 'psychedelic-like' (Carhart-Harris et al. 2014) or a 'stimulant/ psychedelic hybrid' (Roseman et al. 2014), suggesting that the states it elicits approach, but seldom reach, the full intensity of true psychedelic substances such as psilocybin and LSD. 
The use of psychedelic and psychedelic-like drugs at dance clubs and raves can partly be explained by the overlap in the affective states they afford. In fact, the term 'vibe' first entered into the discourse of dance-music culture via its counter-cultural predecessors, not least via psychedelic counter-culture, in which 'vibrations were imagined to be an invisible linking force between participants, promising to forge bonds through sound that would overcome the hierarchies and antagonisms of hegemonic culture' (Garcia, in press).

Furthermore, the experience of 'ego-dissolution'-'a feeling that one's "self", "ego" or "I" is disintegrating or that the border between one's self and the external world is "dissolving"' (Lebedev et al. 2015: 3137)—is reported in both empirical research with psychedelics and in ethnographic studies of dance-music cultures. This is exemplified in the hyperreal.org quotation at the beginning of this chapter and further elaborated in the section 'Vibe and embodied distribution in clubbing and raving'. In their study (p.106) of psychedelics, Robin Carhart-Harris and colleagues have made connections between primary consciousness and 'oceanic states', citing Freud (1961) and Walter Stace's (1960) descriptions of experiences where 'the complex multiplicity of normal consciousness collapses into a simpler state where a sense of an allencompassing "oneness" with others, the world and/or "God" is felt' (CarhartHarris 2014: 15; italics in original). The overlapping experiences of 'ego dissolution', 'oceanic states', and primary consciousness suggest that an awareness of extended or 'expanded' mind might provide a link between psychedelic and dance-music experiences. 
By administering psychedelic drugs to their research participants in placebocontrolled experiments, Carhart-Harris and colleagues have claimed that they have experimentally induced states of primary consciousness, thus enabling the study of the brain's functional transformations during such embodied awareness. One of their main findings is that psychedelics seem to increase the complexity of the brain's functional organization. From studies using functional magnetic resonance imaging (fMRI), they reported that when psilocybin was administered to their participants, 'cerebral blood flow, BOLD signal, functional connectivity and oscillatory power were decreased in brain regions that are normally highly metabolically active, functionally connected and synchronous/ organized in their activity' (Carhart-Harris et al. 2014: 6). ${ }^{8}$ In other words, the psychedelic brain seems less organized overall than the normal brain. The investigators also found that, following administration of psilocybin, the brain's default mode network (DMN) behaved differently (Carhart-Harris et al. 2012). The DMN is a network of brain regions that are densely connected and functionally correlated when an individual is not consciously focusing on the outside world. ${ }^{9}$ That is, in the absence of a specific task, the brain falls 'by default' into a specific neurofunctional pattern (Greicius et al. 2003). Activity in the DMN has been associated with a number of metacognitive operations that are characteristic of higher-order consciousness, such as self-reflection (Qin and Northoff 2011), theory of mind (Spreng and Grady 2010), and mental time travel (Buckner and Carroll 2007). Carhart-Harris and colleagues (2012) found that during a psychedelic state, the brain exhibited reduced coupling between the medial temporal lobe (MTL) and the DMN-a functional connection that is implicated in normal waking consciousness. In addition, the brain exhibited increased BOLD signal variance in the MTL, consistent with decreased connectivity between the MTL and the DMN. The authors thus proposed that coupling in the DMN-specifically between the DMN and the MTL-is a neural correlate of higher-order consciousness and a sense of self. When these areas are decoupled, such as during the psychedelic state, primary consciousness is more dominant, and the sense of ego is attenuated (Carhart-Harris et al. 2014).

(p.107) Schartner et al. (2017) provide further empirical support for the increased complexity of the brain's functional organization during the psychedelic state. Using magnetoencephalography (MEG), the authors showed that the signal diversity, or entropy, of the brain's neurophysiological patterns is increased following the administration of psilocybin, LSD, and ketamine. Importantly, the study participants' subjective reports of the psychedelic experience correlated with the neural signal diversity, suggesting that increased brain entropy is correlated with more intense psychedelic experiences. These results are consistent with the idea that levels of consciousness can be indicated by levels of neural complexity. Carhart-Harris et al. (2014) suggest that the brain is more entropic during states of primary consciousness, and specifically that it involves increased neural criticality and metastability. 
Criticality is a network state that occurs at the transition from an ordered to disordered state, when the system approaches 'the edge of chaos' (Sporns 2010: 268). ${ }^{10}$ Compared to subcritical and supercritical systems, a critical system undergoes the largest and fastest fluctuations, and is the most susceptible to perturbation. Any change to the system will rearrange the system's organization. In the brain, such criticality in the neural networks gives rise to what has been termed 'metastability' in brain dynamics. Metastability in the brain is a sequence of transient states each of which is neither entirely stable nor entirely unstable (Tognoli and Kelso 2014). It describes a balance between neural integration (when neural spiking is synchronized) and segregation (uncoupled spiking), with moments of local synchrony that are never phase-locked. Each metastable state depends on the underlying network's functional relationships, and the transitions between these transient metastable states result in 'switching' between networks. Such complex yet relatively coordinated neural dynamics are thought to facilitate maximal flexibility in how the brain is configured: any change or perturbation can trigger a reorganization of the system. When the brain is close to criticality and metastable, it is thought to provide access to increasingly diverse neural configurations and cognitive states (Kringelbach et al. 2015).

What Carhart-Harris et al. (2014) suggest is that the criticality- and metastability-consistent evidence of increased entropy in the brain could potentially play a part in the subjectively experienced richness, 'randomness', openness, and perceptual and cognitive enhancements associated with the psychedelic state. During normal higher-order consciousness, the brain is subcritical, and can in this way better control how it engages with the environment by having a firmer grasp on sensory inputs, tuning in only to relevant perceptual information. By contrast, in induced primary consciousness as elicited by psychedelics, the brain is supercritical. People on psychedelic drugs can be hypersensitive to even the smallest sensory perturbations, and may be abnormally concerned with a greater (p.108) and more complex array of inputs afforded by the environment. Carhart-Harris's research suggests that this relates to the metastable spatiotemporal dynamics of the brain that psychedelics induce. 
If there are any similarities between the 'oceanic' experiences of primary consciousness (and thus an awareness of extended mind) afforded by psychedelics and those afforded by dancing to house and techno music and getting into the vibe of a dance party, then it is also possible that the brain operates in a similar way during clubbing and raving as in the psychedelic state. In other words, it may be that in addition to already known neural correlates of music-such as rhythm responses in the basal ganglia and motor areas (Chen 2008; Grahn and Brett 2007; Vuust and Roepstorff 2008) and musical pleasure responses in the ventral striatum and orbitofrontal cortex (Blood and Zatorre 2001; Salimpoor et al. 2011; Vuust and Kringelbach 2010)—the increased neural entropy that supports the extended consciousness of psychedelic experiences would also be found during the extended consciousness of clubbing and raving. Feeling at one with the music and the crowd, and the cognitive, social, and affective distribution that people experience in a dance club, may be related to increased neural metastability.

\section{Conclusion}

This chapter has applied affective and social extensions of theories of cognitive distribution to the musical consciousness associated with dancing in house and techno clubs, raves, and dance-music festivals. The vibe of a dance party is an example of 'affective atmosphere': a smooth, surfaceless space where subjectivity is secondary to feeling intensities. This vibe is defined as a cognitively, socially, affectively, bodily, and environmentally distributed phenomenon that has a distinct rhythmic and textural quality, and that cannot be reduced to its individual parts-the music, the DJ, the dancers, the club or rave space, the lights, the temperature, or the drugs. When the vibe reaches a high intensity-when experiences become oceanic, timeless, and ecstatic-it affords a musical state that resembles primary consciousness, in which bodily and felt knowing prevail over reflexive and analytic knowledge. While we do not yet know the neurofunctional architecture of the brain during such intensely pleasurable experiences of dancing, we can draw on other neuroimaging studies in which states of primary consciousness are induced via psychedelic drugs. These studies suggest that primary consciousness is associated with decreased coupling between certain brain networks and increased complexity, criticality and metastability in the brain. A similarly entropic brain could support the cognitive, social, and affective distribution that clubbers and ravers experience when they synchronize their bodies to the beat and to the movements of the dancers around them, and feel at one with the music, the crowd, and the world. 
We might predict, however, that without psychoactive drugs, the metastability of the club dancers' neural networks would be reduced. If we accept that drugs such as MDMA-or indeed psychedelic substances such as psilocybin and LSDare used to enhance the oceanic experiences that clubbers and ravers can only experience fleetingly (p.109) without drugs, then we can hypothesize that the sober experience is a less prolonged and less intense version of the drugged experience. Furthermore, there may be important differences between the experience of a musical vibe and primary consciousness understood as a more general state of musical listening. Listening is never completely 'passive' and usually involves some level —albeit often covert-of active engagement and multisensory perception. Nonetheless, it seems that the active and explicitly multisensory state of clubbing and raving can make these aspects of musical experience more intense than other more motionless and solitary ways of listening to music.

Finally, what makes the vibe stand out from other forms of primary musical consciousness is the explicit manifestation and celebration of cognitive, social, and affective distribution-leading to those detailed descriptions of feelings of oneness that are found on hyperreal.org. With 'everyday' listening, we may be less conscious of how our minds and bodies are intertwined with the music and our surroundings-in the same way that conscious awareness of the vibe's distributed character is typically not a narrative type of awareness during the experience itself. In the act of dancing and raving, at least to the seasoned raver, the vibe is an embodied, felt kind of knowing that is only recounted in retrospect -only represented in higher consciousness once the raver has come off the dance floor or gone home from the party. Ultimately, the socio-affective distribution of the vibe relies on both immediate, primary consciousness as well as narrative, higher consciousness. It is the vibe as felt in the 'smooth' moment, and as reflected on in those 'striated' face-to-face and online discussions, that sustains dance music cultures as social and affective communities.

\section{References}

Bibliography references:

Becker, J.O. (2004). Deep Listeners: Music, Emotion, and Trancing. Bloomington, IN: Indiana University Press.

Bedi, G., Phan, K.L., Angstadt, M., and De Wit, H. (2009). Effects of MDMA on sociability and neural response to social threat and social reward.

Psychopharmacology, 207(1), 73-83.

Blood, A.J. and Zatorre, R.J. (2001). Intensely pleasurable responses to music correlate with activity in brain regions implicated with reward and emotion. Proceedings of the National Academy of Sciences, 98(20), 11818-23. 
Feeling at one: Socio-affective distribution, vibe, and dance-music consciousness

Buckner, R.L. and Carroll, D.C. (2007). Self-projection and the brain. Trends in Cognitive Sciences, 11(2), 49-57.

Carhart-Harris, R.L., Erritzoe, D., Williams, T., Stone, J.M., Reed, L.J., Colasanti, A., Tyacke, R.J., Leech, R., Malizia, A.L., Murphy, K., Hobden, P., Evans, J., Feilding, A., Wise, R.G., and Nutt, D.J. (2012). Neural correlates of the psychedelic state as determined by fMRI studies with psilocybin. Proceedings of the National Academy of Sciences, 109(6), 2138-43.

Carhart-Harris, R.L., Carhart-Harris, R.L., Leech, R., Hellyer, P.J., Shanahan, M., Feilding, A., Tagliazucchi, E., Chialvo, D.R., and Nutt, D. (2014). The entropic brain: A theory of conscious states informed by neuroimaging research with psychedelic drugs. Frontiers in Human Neuroscience, $\mathbf{8}$.

Chen, J., Penhune, V.B., and Zatorre, R.J. (2008). Listening to musical rhythms recruits motor regions of the brain. Cerebral Cortex, 18(12), 2844-54.

Chialvo, D.R. (2010). Emergent complex neural dynamics. Nature Physics, 6(10), 744-50.

(p.110) Clark, A. (2008). Supersizing the Mind: Embodiment, Action, and Cognitive Extension. New York, NY: Oxford University Press.

Clark, A. (2009). Spreading the joy? Why the machinery of consciousness is (probably) still in the head. Mind, 118(472), 963-93.

Clark, A. and Chalmers, D. (1998). The extended mind. Analysis, 58(1), 7-19.

Clarke, E.F. (2011). Music perception and musical consciousness. In D. Clarke and E.F. Clarke (eds.), Music and Consciousness: Philosophical, Psychological, and Cultural Perspectives (pp. 193-213). Oxford: Oxford University Press.

Clarke, E.F. (2014). Lost and found in music: Music, consciousness and subjectivity. Musicae Scientiae, 18(3), 354-68.

Damasio, A.R. (1999). The Feeling of What Happens: Body and Emotion in the Making of Consciousness. New York, NY: Harcourt Brace.

Deleuze, G. and Guattari, F. (1987). A Thousand Plateaus: Capitalism and Schizophrenia. Minneapolis, MN: University of Minnesota Press.

DeNora, T. (2000). Music in Everyday Life. Cambridge: Cambridge University Press.

Dumont, G. and Verkes, R. (2006). A review of acute effects of 3, 4methylenedioxymethamphetamine in healthy volunteers. Journal of Psychopharmacology, 20(2), 176-87. 
Feeling at one: Socio-affective distribution, vibe, and dance-music consciousness

Durkheim, E. (1995). The Elementary Forms of Religious Life, trans. K.E. Fields. New York, NY: Free Press. [Originally published as Les formes elémentaires de la vie réligieuse: Le système totémique en Australie. Paris: F. Alcan, 1912.]

Edelman, G.M. (1989). The Remembered Present: A Biological Theory of Consciousness. New York, NY: Basic Books.

Fikentscher, K. (2000). 'You Better Work!' Underground Dance Music in New York City. Middletown, CT: Wesleyan University Press.

Freud, S. (1961). Civilisation and its Discontents, trans. and ed. J. Strachey. London: Hogarth Press. [Originally published as Das Unbehagen in der Kultur. Vienna: Internationaler Psychoanalytischer Verlag, 1930.]

Gallagher, S. (2011). The overextended mind. Versus: Quaderni di studi semiotici, 112-113, 57-68.

Gallagher, S. (2013). The socially extended mind. Cognitive Systems Research, 25-26, 4-12.

Garcia, L.-M. (2011). 'Can You Feel It, Too? Intimacy and Affect at Electronic Dance Music Events in Paris, Chicago and Berlin.' Unpublished doctoral dissertation, University of Chicago.

Garcia, L.-M. (2015). Beats, flesh, and grain: Sonic tactility and affect in electronic dance music. Sound Studies, 1(1), 59-76.

Garcia, L.-M. (2016). Perspective: What happened to the '24-hour city'? Crack Magazine, 1 September 2017. http://crackmagazine.net/opinion/opinion/ perspective-happened-24-hour-city/

Garcia, L.-M. (in press). Feeling the vibe: Sound, vibration, and affective attunement in electronic dance music scenes. Ethnomusicology Forum.

Gilbert, J. and Pearson, E. (1999). Discographies: Dance Music, Culture and the Politics of Sound. London: Routledge.

Gilmour, C. (2016). The raver's return: How underground parties are making a comeback. The Independent, 30 September 2016. http://www.independent.co.uk/ arts-entertainment/the-ravers-return-how-underground-parties-are-making-acomeback-a7036131.html

Grahn, J.A. and Brett, M. (2007). Rhythm and beat perception in motor areas of the brain. Journal of Cognitive Neuroscience, 19(5), 893-906.

Greicius, M.D., Krasnow, B., Reiss, A.L., and Menon, V. (2003). Functional connectivity in the resting brain: A network analysis of the default mode hypothesis. Proceedings of the National Academy of Sciences, 100(1), 253-8. 
Feeling at one: Socio-affective distribution, vibe, and dance-music consciousness

(p.111) Griffiths, R.R., Richards, W.A., McCann, U., and Jesse, R. (2006). Psilocybin can occasion mystical-type experiences having substantial and sustained personal meaning and spiritual significance. Psychopharmacology, 187(3), 268-83.

Hove, M.J., Hove, M.J., Stelzer, J., Nierhaus, T., Thiel, S.D., Gundlach, C., Margulies, D.S., Van Dijk, K.R., Turner, R., Keller, P.E., and Merker, B. (2016). Brain network reconfiguration and perceptual decoupling during an absorptive state of consciousness. Cerebral Cortex, 26(7), 3116-24.

hyperreal.org (30 September 2016). http://hyperreal.org/ (accessed 28 January 2019).

Jones, M.R. (2009). Musical time. In S. Hallan, I. Cross, and M. Thaut (eds.), The Oxford Handbook of Music Psychology (pp. 81-92). New York, NY: Oxford University Press.

Jordan, T. (1995). Collective bodies: Raving and the politics of Gilles Deleuze and Felix Guattari. Body \& Society, 1(1), 125-44.

Karwowski, H. (2016). Lightning struck at Berghain this weekend-and this is what the artists are saying. Monument, 30 September 2016. https://mnmt.no/ blog/lightning-strikes-berghain/

Kringelbach, M.L., McIntosh, A.R., Ritter, P., Jirsa, V.K., and Deco, G. (2015). The rediscovery of slowness: Exploring the timing of cognition. Trends in Cognitive Sciences, 19(10), 616-28.

Krueger, J. (2013). Affordances and the musically extended mind. Frontiers in Psychology, 4(1003), 1-13. http://dx.doi.org/10.3389/fpsyg.2013.01003

Krueger, J. (2015). The affective 'we': Self-regulation and shared emotions. In T. Szanto and D. Moran (eds.), The Phenomenology of Sociality: Discovering the 'We' (pp. 263-77). London: Routledge.

Lawrence, T. (2011). Disco and the queering of the dance floor. Cultural Studies, 25(2), 230-43.

Lebedev, A.V., Lövdén, M., Rosenthal, G., Feilding, A., Nutt, D., and CarhartHarris, R. (2015). Finding the self by loosing the self: Neural correlates of egodissolution under psilocybin. Human Brain Mapping, 36(8), 3137-53.

Malbon, B. (2002). Clubbing: Dancing, Ecstasy, Vitality. London: Routledge. 
Feeling at one: Socio-affective distribution, vibe, and dance-music consciousness

Oltermann, P. (2016). High culture club: Berghain secures same tax status as Berlin concert venues. The Guardian, 30 September 2016. https://

www.theguardian.com/music/2016/sep/12/berlins-berghain-nightclub-classed-asculturally-significant-venue

Palamar, J.J., Acosta, P., Ompad, D.C., and Cleland, C.M. (2017). Self-reported ecstacy/MDMA/'molly' use in a sample of nightclub and dance-festival attendees in New York City. Substance Use \& Missue, 52(1), 82-91.

Phillips-Silver, J., Aktipis, A.C., and Bryant, G. (2010). The ecology of entrainment: Foundations of coordinated rhythmic movement. Music Perception, 28(1), 3-14.

Qin, P. and Northoff, G. (2011). How is our self related to midline regions and the default-mode network? Neuroimage, 57(3), 1221-33.

Riedel, F. (2015). Music as atmosphere. Lines of becoming in congregational worship. Lebenswelt: Aesthetics and Philosophy of Experience, 6, 80-111.

Rief, S. (2011). Club Cultures: Boundaries, Identities and Otherness. London: Routledge.

Rietveld, H. (1993). Living the dream. In S. Redhead (ed.), Rave Off: Politics and Deviance in Contemporary Youth Culture (pp. 41-78). Aldershot: Avebury.

Roseman, L., Leech, R., Feilding, A., Nutt, D., and Carhart-Harris, R.L. (2014). The effects of psilocybin and MDMA on between-network resting state functional connectivity in healthy volunteers. Frontiers in Human Neuroscience, 8(204), 1 11. https://doi.org/10.3389/fnhum.2014.00204

Rowlands, M. (2010). The New Science of the Mind: From Extended Mind to Embodied Phenomenology. Cambridge, MA: MIT Press.

(p.112) Salimpoor, V.N., Beovoy, M., Larcher, K., Dagher, A., and Zatorre, R.J. (2011). Anatomically distinct dopamine release during anticipation and experience of peak emotion to music. Nature, Neuroscience, 14(2), 257-62.

Schartner, M.M., Carhart-Harris, R.L., Barrett, A.B., Seth, A.K., and Muthukumaraswamy, S.D. (2017). Increased spontaneous MEG signal diversity for psychoactive doses of ketamine, LSD and psilocybin. Scientific Reports, 7(46421), 1-12.

Schmitz, H., Müllan, R.O., and Slaby, J. (2011). Emotions outside the box-the new phenomenology of feeling and corporeality. Phenomenology and the Cognitive Sciences, 10(2), 241-59. 
Feeling at one: Socio-affective distribution, vibe, and dance-music consciousness

Slaby, J. (2014). Emotions and the extended mind. In C. Von Scheve and M. Salmela (eds.), Collective Emotions: Perspectives from Psychology, Philosophy, and Sociology (pp. 32-46). Oxford: Oxford University Press.

Sommer, S.R. (2001). 'C'mon to my house': Underground-House dancing. Dance Research Journal, 33(2), 72-86.

Sporns, O. (2010). Networks of the Brain. Cambridge, MA: MIT Press.

Spreng, R.N. and Grady, C.L. (2010). Patterns of brain activity supporting autobiographical memory, prospection, and theory of mind, and their relationship to the default mode network. Journal of Cognitive Neuroscience, 22(6), 1112-23.

St John, G. (2008). Trance tribes and dance vibes: Victor Turner and electronic dance music culture. In G. St John (ed.), Victor Turner and Contemporary Cultural Performance (pp. 149-73). New York, NY: Berghahn Books.

Stace, W.T. (1960). Mysticism and Philosophy. London: Macmillan.

Stephan, A., Walter, S., and Wilutzky, W. (2013). Emotions beyond brain and body. Philosophical Psychology, 27(1), 65-81.

Thompson, E. (2010). Mind in Life: Biology, Phenomenology and the Sciences of Mind. Cambridge, MA: Belknap Harvard University Press.

Thornton, S. (1995). Club Cultures: Music, Media and Subcultural Capital. Cambridge: Polity Press.

Tognoli, E. and Kelso, J.S. (2014). The metastable brain. Neuron 81(1), 35-48.

Trost, V., Labbé, C., and Grandjean, D. (2017). Rhythmic entrainment as a musical affect induction mechanism. Neuropsychologia, 26, 96-110.

Von Scheve, C. and Ismer, S. (2013). Towards a theory of collective emotions. Emotion Review, 5(4), 406-13.

Von Scheve, C. and Salmela, M. (2014). Collective emotions: An introduction. In C. Von Scheve and M. Salmela (eds.), Collective Emotions: Perspectives from Psychology, Philosophy and Sociology, pp. xiii-xxiv. Oxford: Oxford University Press.

Vuust, P. and Kringelbach, M.L. (2010). The pleasure of making sense of music. Interdisciplinary Science Reviews, 35(2), 166-92.

Vuust, P. and Roepstorff, A. (2008). Listen up! Polyrhythms in brain and music. Cognitive Semiotics, 3, 134-58. 
Feeling at one: Socio-affective distribution, vibe, and dance-music consciousness

Ward, D. (2012). Enjoying the spread: Conscious externalism reconsidered. Mind, 121(483), 731-51.

West, P.M. (1996). http://hyperreal.org/raves/spirit/vibe/Vibe_Experience.html

Windt, J. (2017). Predictive brains, dreaming selves, sleeping bodies: How the analysis of dream movement can inform a theory of self- and world-simulation in dreams. Synthese. http://dx.doi.org/10.1007/s11229-017-1525-6

Witek, M.A.G. (2017). Filling in: Syncopation, pleasure and distributed embodiment in groove. Music Analysis, 36(1), 138-60.

Notes:

$\left({ }^{1}\right)$ Clark and Chalmers formally state their 'parity principle' as follows: 'If, as we confront some task, a part of the world functions as a process which, were it done in the head, we would have no hesitation in recognizing as part of the cognitive process, then that part of the world is part of the cognitive process' (Clark and Chalmers 1998: 8).

$\left({ }^{2}\right)$ According to Riedel, the notions of smooth and striated space were borrowed by Deleuze and Guattari from Pierre Boulez (Riedel 2015: 102).

$\left({ }^{3}\right)$ Although some have pointed out the religious, specifically tribal and shamanistic, aspects of dance-music culture (St John 2008).

$\left({ }^{4}\right)$ Clarke uses Edelman's (1989) terminology of 'core' and 'extended' consciousness to refer to this same distinction. However, in order to avoid confusion between Edelman's 'extended consciousness' and 'extended mind' as discussed in this chapter, I will use Damasio's terms 'primary' and 'higher-order' consciousness.

$\left({ }^{5}\right)$ This contrasts with the 'deep listeners' that Judith Becker (2004) describes in her work.

( $\left.{ }^{6}\right)$ However, see Hove et al. 2016 for a study on functional connectivity-the extent to which neural activity is correlated across areas of the brain-during trance induced by repetitive drumming.

( $\left.{ }^{7}\right)$ They are classified as C-class drugs and are thus illegal in most countries.

$\left(^{8}\right)$ BOLD stands for 'blood-oxygenated-level-dependent', and is used as an indicator of brain metabolism and thus neural activity.

$\left({ }^{9}\right)$ The DMN has its 'hubs' in the posterior cingulate cortex, medial prefrontal cortex, and angular gyrus. 
Feeling at one: Socio-affective distribution, vibe, and dance-music consciousness

$\left({ }^{10}\right)$ A heated magnet provides an example of a critical system (Chialvo 2010). At low temperatures, the system is ordered, exhibiting directionally aligned electron spins. As the system is heated, the spins become increasingly disorganized, resulting in decreased magnetism, until the critical temperature is reached, beyond which the magnetism disappears due to the mutual cancelling out of the disordered spins. 